\title{
Correction to: The antiangiogenic activities of ethanolic crude extracts of four Salvia species
}

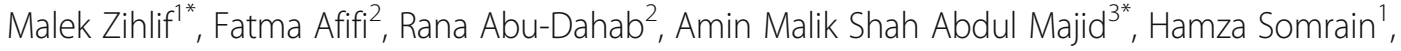
Mohanad M. Saleh ${ }^{1}$, Zeyad D. Nassar ${ }^{3,4}$ and Randa Naffa ${ }^{5}$

\section{Correction}

After the publication [1] it came to the attention of the authors that one of the co-authors was incorrectly included as Hamza Somrain. The correct spelling is as follows: Hamzeh Sumrein.

\begin{abstract}
Author details
'Department of Pharmacology, Faculty of Medicine, The University of Jordan, Amman 11942, Jordan. ${ }^{2}$ Department of Pharmaceutical Sciences, Faculty of Pharmacy, The University of Jordan, Amman, Jordan. ${ }^{3}$ Department of Pharmacology, School of Pharmaceutical Sciences, Universiti Sains Malaysia, 11800 Minden, Penang, Malaysia. ${ }^{4}$ University of Queensland, School of Pharmacy, 20 Cornwall Street, Woolloongabba, QLD 4102, Australia. 5 Department of Physiology and Biochemistry, Faculty of Medicine, The University of Jordan, Amman 11942, Jordan.
\end{abstract}

Received: 5 February 2018 Accepted: 7 February 2018

Published online: 16 February 2018

\section{Reference}

1. Zihlif $\mathrm{M}$, et al. The antiangiogenic activities of ethanolic crude extracts of four Salvia species. BMC Complement Altern Med. 2013;13:358. https://doi. org/10.1186/1472-6882-13-358.

\footnotetext{
*Correspondence: M.zihlif@ju.edu.jo; aminmalikshah@gmail.com

'Department of Pharmacology, Faculty of Medicine, The University of Jordan, Amman 11942, Jordan

${ }^{3}$ Department of Pharmacology, School of Pharmaceutical Sciences, Universiti Sains Malaysia, 11800 Minden, Penang, Malaysia
} 